\section{BA Institute of \\ YK Business Administration \\ 六下 \\ Karachi \\ Leadership and Ideas for Tomorrow}

Business Review

Volume 3 Issue 1 January-June 2008

$1-1-2008$

\title{
Gradient-based edge detection on a hexagonal structure
}

\author{
Xiangjian He \\ University of Technology, Sydney, Australia \\ Qiang Wu \\ University of Technology, Sydney, Australia \\ Tom Hintz \\ University of Technology, Sydney, Australia \\ Wenjing Jia \\ University of Technology, Sydney, Australia
}

Follow this and additional works at: https://ir.iba.edu.pk/businessreview

Part of the Computer Sciences Commons

c) (i)

This work is licensed under a Creative Commons Attribution 4.0 International License.

\section{Recommended Citation}

He, X., Wu, Q., Hintz, T., \& Jia, W. (2008). Gradient-based edge detection on a hexagonal structure. Business Review, 3(1), 133-144. Retrieved from https://doi.org/10.54784/1990-6587.1137

This article is brought to you by iRepository for open access under the Creative Commons Attribution 4.0 License and is available at https://ir.iba.edu.pk/businessreview/vol3/iss1/11. For more information, please contact irepository@iba.edu.pk. 


\title{
DISCUSSION
}

\section{Gradient-Based Edge Detection on a Hexagonal Structure}

\author{
Xiangjian He \\ University of Technology, Sydney, Australia \\ Qiang Wu \\ University of Technology, Sydney, Australia \\ Tom Hintz \\ University of Technology, Sydney, Australia \\ Wenjing Jia \\ University of Technology, Sydney, Australia
}

\begin{abstract}
Gradient-based edge detection is a straightforward method to identify the edge points in the original grey-level image. It is intuitive that in the human vision system the edge points always appear where the grey-level value is greatly changed. Spiral Architecture is a relatively new image data structure that is inspired from anatomical considerations of the primate's vision. In Spiral Architecture, each image is represented as a collection of hexagonal pixels. Edge detection on Spiral Architecture has features of fast computation and accurate localization. In this paper, we review the gradient-based edge detection algorithms on Spiral Architecture. An edge point is defined as a hexagonal pixel at which the magnitude of the gradient of brightness function assumes a local maximum.
\end{abstract}

Keywords: Spiral Architecture, Edge Detection, Triple-Diagonal-Gradient, Bilateral Filter, Prewitt Masks, Sobel Operators, Hexagonal Image Structure

\section{Introduction}

Computer vision involves compositions of picture elements (pixels) into edges, edges into object contours and object contours into scenes. The determination of edges depends on detection of edge points (pixels) of a 3-D physical object in a 2-D image. This first step in the process is critical to the functioning of machine vision. As the success of subsequent steps are sensitive to the quality of results at this step, the performance of higher level processes such as extraction of object contours and object recognition relies heavily on the complete and correct determination of edges [1]. Edges contain major image information and need only a small amount of memory storage space compared to the original image. Hence, edge detection simplifies images and thus facilitates image analysis and interpretation [2]. 
Edge detection is based on the relationship a pixel has with its neighbours. It extracts and localizes points (pixels) around which a large change in image brightness has occurred. A pixel is unsuitable to be recorded as an edge if the brightness around a pixel is similar (or close). Otherwise, the pixel may represent an edge.

During the last three decades, many algorithms have been developed for edge detection, among which the most important ones are the Marr-Hildreth method [3] based on detecting zero crossings at the output of Laplacian-Gaussian operators of different widths, Haralick's facet model [4] based method that uses the zero crossings of a second directional derivative of Gaussian edge operator, and computational approach [5] to edge detection by formulating the task as a numerical optimization problem.

Since 1996, there have been many papers on edge detection based on Spiral Architecture. Spiral Architecture described by Sheridan [6] is a new data structure for computer vision. The image is represented by a collection of hexagons of the same size (in contrast with the traditional rectangular representation). The importance of the hexagonal representation is that it possesses special computational features that are pertinent to the vision process. In [7], edge detection using edge focusing technique was proposed. This method starts from the edge detection of a blurred image with a large scale of Gaussian filter. The scale is gradually decreased for finer images. The final edge map is recorded when it will no longer change the edge map with any smaller scale. The second edge detection method proposed [8] and [9] applied a bilateral filter rather than a Gaussian filter to remove image noise. The bilateral filter combines a domain filter like Gaussian filter with a range filter. A range filter gives more weights to those neighbouring pixels with light intensity that is more similar to the reference pixel value. This method has been proved being more efficient for suppressing image noise for edge detection. Another method for edge detection on Spiral Architecture, as shown in [10] and [11], was based on triplediagonal gradient. The gradient of grey-level function was defined as a combination of three vectors in three diagonal directions of hexagonal image structure. Note that an edge point is a pixel at which the gradient magnitude assumes a local maximum. Hence, this method is a more accurate detection mechanism where the gradient is implemented in a more accurate way in the discrete image space.

In this paper, we will review the above-mentioned edge detection methods on Spiral Architecture. We will also list the problems for future research work.

\section{Spiral Architecture}

In Spiral Architecture, an image is represented as a collection of hexagonal pixels. Each pixel has only six neighbouring pixels with the same distance to it. Each pixel is identified by a number of base 7 called a spiral address. The numbered (or addressed) hexagons form the cluster of size $7^{\mathrm{n}}$, where $\mathrm{n}$ is a positive integer. These 
hexagons starting from address 0 towards address $7^{\mathrm{n}}$ tile the plane in a recursive modular manner along a spiral-like curve. As an example, a cluster with size of $7^{2}$ and the corresponding spiral addresses are shown in Figure 1.

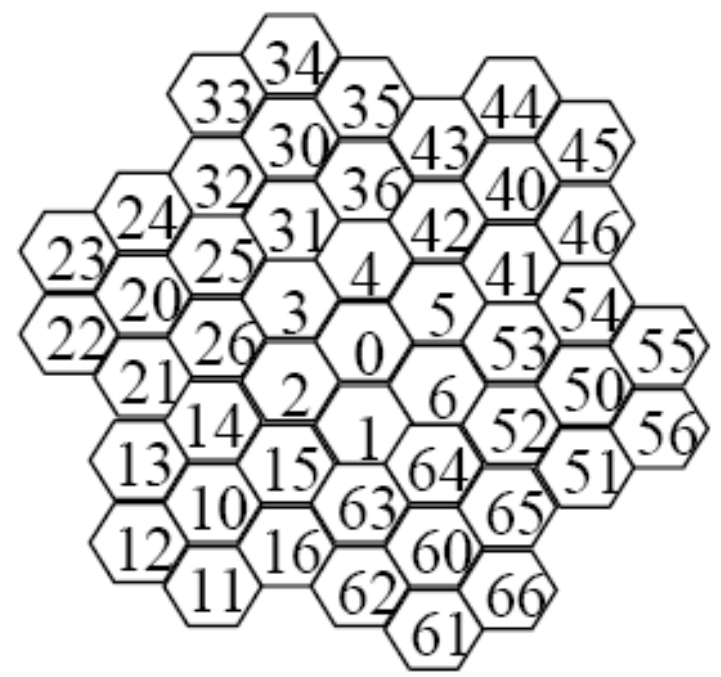

Figure 1. A collection of 49 hexagonal pixels.

The distribution of cones on the retina (see Figure 2) provides the basis of the Spiral Architecture. In the case of the human eye, these elements would represent the relative positions of the rods and cones on the retina.

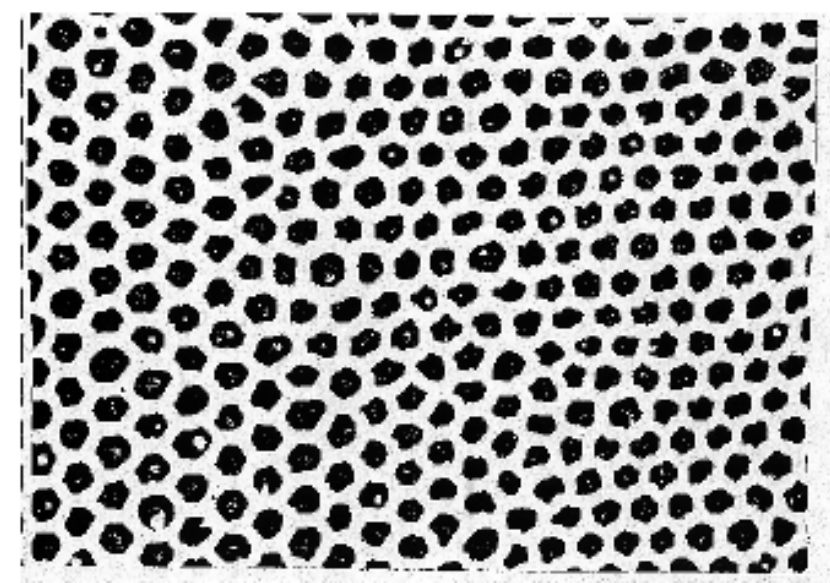

Figure 2. Distribution of cones on the retina. 
Spiral Architecture possesses some geometric and algebraic properties, which are very useful and can be interpreted in terms of a mathematical object, Euclidean ring (Refer to [6] for details). Two algebraic operations have been defined on Spiral Architecture based on spiral addresses. They are Spiral Addition and Spiral Multiplication. These two operations correspond to two transformations on Spiral Architecture, which are translation and rotation with a scaling.

\section{Edge Focusing Edge Detection}

Gaussian Multi-scale Theory is one of the best understood multi-resolution techniques available to the computer vision community [12]. Edge detection using Gaussian Multi-scale Theory ensures not only good performance of detection but also accurate localization of edge points.

Let $f: \Re^{2} \rightarrow \Re$ be a brightness function of an image which maps the coordinates of a pixel, $(x, y)$ to a value in light intensities. Let $g: \mathfrak{R}^{2} \times(0,+\infty) \rightarrow \mathfrak{R}$ be the Gaussian kernel

$$
g(x, y ; t)=\frac{1}{2 \pi t} e^{-\frac{\left(x^{2}+y^{2}\right)}{2 t}} .
$$

Then the Gaussian scale-representation of the original image at scale $t$ is

$$
\begin{aligned}
L(x, y ; t) & =g(x, y ; t) * f(x, y) \\
& =\iint_{\sigma} f(u, v) \frac{1}{2 \pi t} e^{-\frac{(x-u)^{2}+(y-v)^{2}}{2 t}} d u d v
\end{aligned}
$$

when $t \in(0, \infty)$, and $L(x, y ; 0)=f(x, y)$.

Scale-space representation is used to suppress and remove unnecessary and disturbing details so that later stage processing tasks can be simplified.

Edge point from a continuous grey-level image represented as $L(x, y ; t)$ for given $t$ is defined as a pixel at which the gradient magnitude of $L(x, y ; t)$ assumes a local maximum in the gradient direction.

The edge focusing technique starts with a blurred image with a large Gaussian scale (or coarse resolution). An edge map consisting edge points is obtained through this strong blurring. The next step is to gradually focus these edge points by continuously decreasing the scale (or resolution). This method compares the edge maps at different levels of resolution (or with different scales) to decide whether to repeat the detection process with a smaller scale or terminate the process. 


\section{Edge Detection Using Bilateral Filter}

Recall that a bilateral filter is defined by the combination of a domain filter and a range filter. Let $a_{0}$ be a reference pixel and $a_{1}, a_{2}, a_{3}, a_{4}, a_{5}, a_{6}$ be the six neighbouring pixels of $a_{0}$. Then, the range filter is defined by

$$
r(a)=e^{-\frac{\left[f\left(a_{i}\right)-f\left(a_{0}\right)\right]^{2}}{2 \sigma_{r}^{2}}}, i=0,1,2, . .6
$$

where $\sigma_{r}$ is the standard deviation of intensity value distribution. The domain filter defined by

$$
g(a)=e^{-\frac{\left[d\left(a_{i}, a_{0}\right)\right]^{2}}{2 \sigma_{d}{ }^{2}}}, i=0,1,2, . .6
$$

where $d\left(a_{i}, a_{0}\right)$ is the Euclidean distance between $a_{i}$ and $a_{0}$, and $\sigma_{d}$ is the standard deviation in spatial distribution.

Image noise is filtered through the convolution of the image brightness function with the domain and range filters. Through the domain filter, details can be gradually smoothed. Through the range filter, blurring effects across the edges can be reduced and edges can be preserved better than using domain filtering alone. Application of the new bilateral smoothing filter produces, for each pixel in the image, a weighted average such that each pixel contributes more significantly to the resulting grey value of the pixel than all its neighbouring pixels. Meanwhile, the pixels with more similar intensity values or closer to the reference pixel contribute more than those with more different values or further away.

Smoothing using the bilateral filter clearly reduces the blurring effect introduced by smoothing to images than Gaussian domain smoothing alone. It implies that edge information would be better retained with less averaging across the kernel under bilateral smoothing. This can be seen in the experimental results shown in Section 6 below.

After image noise is filtered, Sobel operator defined on SA as shown in Figure 3 is applied. Using the Sobel operator, the pixels' gradient values are obtained. At the same time, the edge length and direction can are determined.

Edge pixels on SA are fetched using the method similar to Canny edge detection method worked on the traditional square structure. Candidate edge points are located first using local non-minimal suppression [5, 9]. In order to recover missing weak edge points and eliminate false edge points, two edge strength thresholds are set to examine all the candidate edge points. Those candidate edge points whose grey 
levels are below the lower threshold are marked as non-edge points. For each candidate edge point whose grey levels is above the lower threshold, if it can be connected to a edge point whose edge strength is above the higher threshold through a chain of edge points, it is then marked as an edge point.

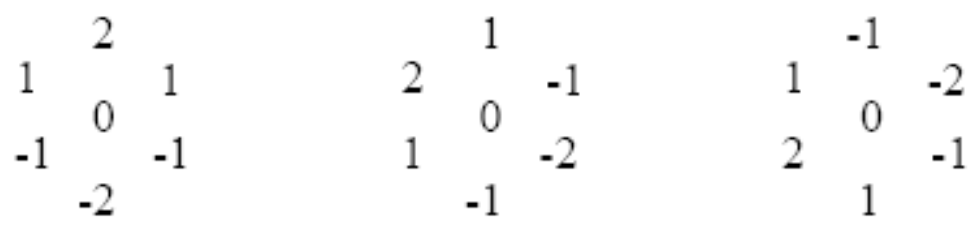

Figure 3. Sobel operators on hexagonal structure.

\section{Using Triple-diagonal Gradient for Edge Detection}

In this method, three gradient components on three diagonal directions instead of one approximated gradient direction are computed at each pixel.

Let $L_{\bar{v}}$ be the gradient of the brightness function $L$ at a given reference point and $G_{i}$ ( $i \in\{1,2,3\}$ ) be the three gradient components in the three diagonal directions respectively for a given reference point as shown in Figure 4 . We call the three gradient components triple-diagonal gradient components. In the real Spiral Architecture, the distance between the reference point and any of its neighbouring point is same. Without loss of generality, we assume that the distance is 1 . It is then easy to see that

$$
L_{\bar{v}}=G_{1}(1,0)+G_{2}(1 / 2, \sqrt{3} / 2)+G_{3}(1 / 2,-\sqrt{3} / 2),
$$

where the three vectors corresponding to the three diagonal directions as shown in Figure 5.
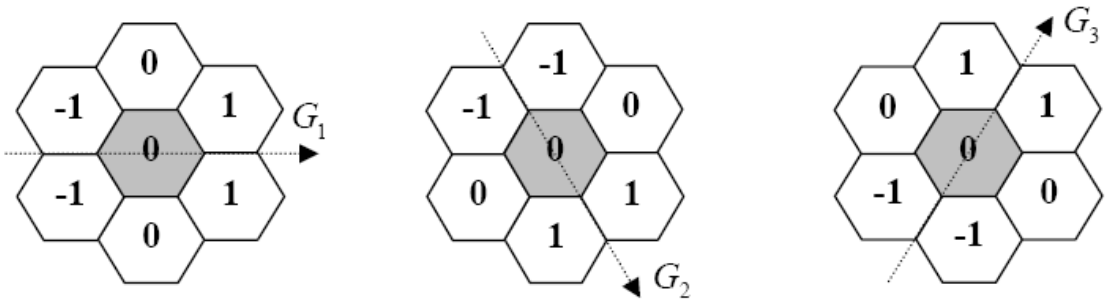

Figure 4. Prewitt Masks in three diagonal directions on hexagonal structure. 

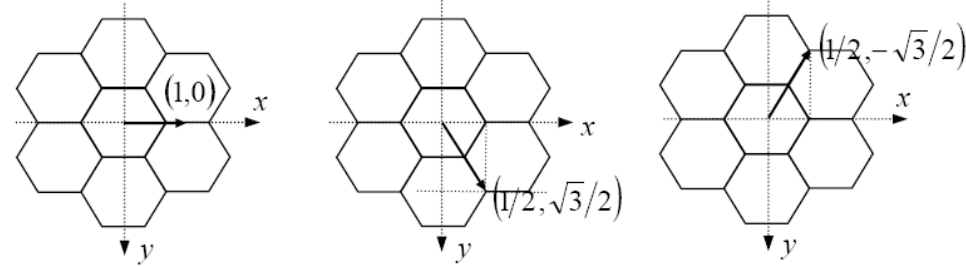

Figure 5. Three unit vectors in three diagonal directions.

The three gradient components are computed using the Prewitt Masks defined in Figure 4. The gradient magnitude at each hexagonal pixel can hence be computed using

$$
\sqrt{\left(\frac{2 G_{1}+G_{2}+G_{3}}{2}\right)^{2}+\left(\frac{\sqrt{3}\left(G_{2}-G_{3}\right)}{2}\right)^{2}} .
$$

\section{Experimental results}

In this section, we show the experimental results using the three different edge detection methods described above. A toy duck and the Lena image represented on Spiral Architecture with 256 grey levels are used as the original image for edge detection.

Figure 6 shows three images processed by different filtering operations on different image architecture. Figure 7 shows the edge maps obtained from the three filtered images accordingly. As can be seen in Figure 6, Gaussian operator works better on SA than square structure, and the bilateral operator shows a better filtering result than Gaussian filter. The bilateral filtering process enhances the major edge information and weakens other pixels. It is obvious that after Gaussian filtering edge map obtained on SA is clearer than the one obtained on square architecture. This is mainly due to the uniformly connected. Moreover, one more Sobel operator defined on SA than the two operators on square architecture improves the detection accuracy. The bilateral filtering further improves the edge map quality because it enhances major edge information while suppressing image noise and trivial edge pixels.

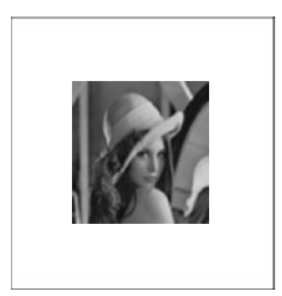

(a) Gaussian filtering on square architecture

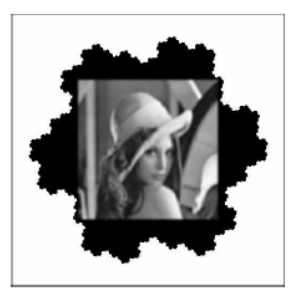

(b) Gaussian filtering on hexagonal architecture

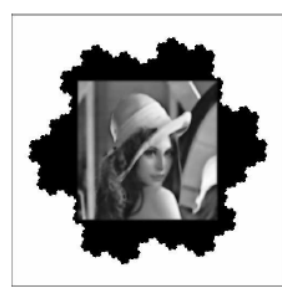

(c) Bi-lateral filtering on hexagonal architecture

Figure6. Results of image filtering. 


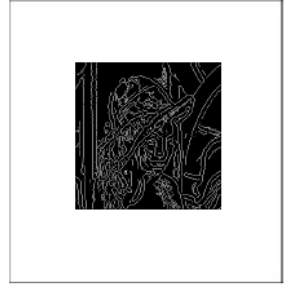

(a) Edge map after Gaussian

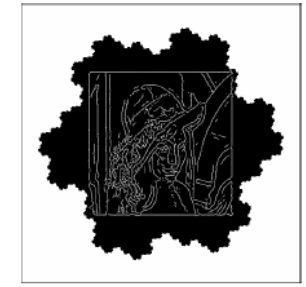

(b) Edge map after Gaussian

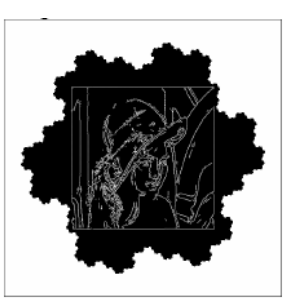

(c) Edge map after bi-lateral

Figure 7. Edge maps of the filtered images shown in Figure 6.

Figure 8 shows the gradient edge maps obtained on square structure and on Spiral Architecture using the Triple-Diagonal-Gradient approach. From the results, we can see that, if we focus on the edge map of the circular edges, we can find that a thinner edge map can be acquired on hexagonal-based processing. This is mainly due to the good performance of the triple-diagonal detectors on hexagonal images that are able to highlight the strongest response not only on horizontal and vertical directions, but also on the other two diagonal directions. In order to obtain the similar performance on square images, we need another two additional Prewitt masks in diagonal and inverse diagonal directions. In hexagonal images, we only need total three Prewitt masks.

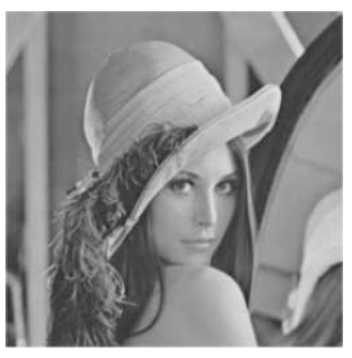

(a)

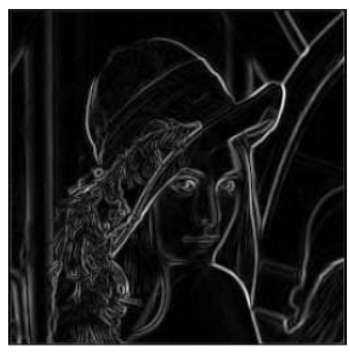

(b)

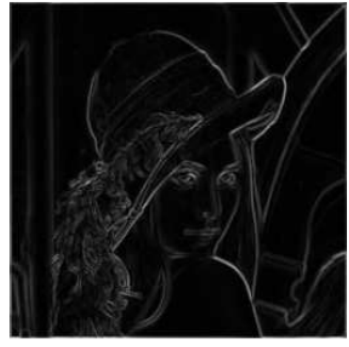

(c)

Figure 8. Gradient-based edge maps of Lena image. (a) Original image, (b) the gradient edge map implemented on square architecture, and (c) gradient map implemented on SA

In order to compare among the edge focusing technique, the bilateral filtering method and the triple-diagonal-gradient approach, a toy duck as shown in Figure 9 is used. 


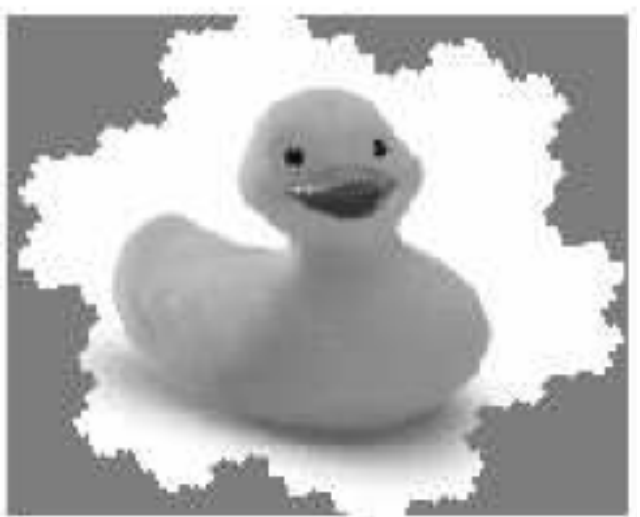

Figure 9. Image of a toy duck represented on SA.

The edge detection results are displayed in Figure 10.

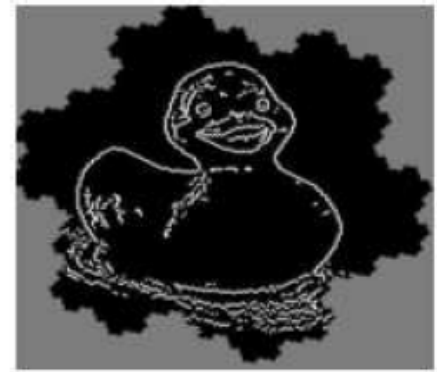

(a)

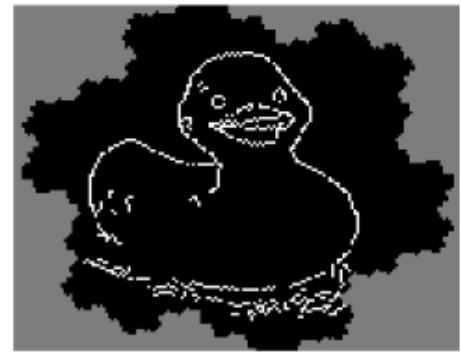

(c)

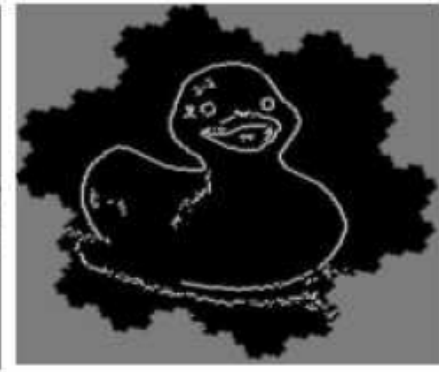

(b)

Figure 10. (a) edge map using edge focusing technique, (b) edge map using bilateral filter, (c) edge map using triple-diagonal gradient

Comparison between Figure 10(a) and Figure 10(b) clearly demonstrates better edgepreserving quality of the bilateral smoothing than Gaussian smoothing. Contour of the mouth is again more complete in the final edge map when bilateral smoothing was applied than it is in the edge map obtained after Gaussian smoothing only. 
Comparison between Figure 10(b) and Figure 10(c) shows that the edge noise has been better filtered out using bilateral smoothing than using triple-diagonal gradient. On the other hand, the use of triple-diagonal gradient gives us the sharpest and clearest edge map among the three methods.

\section{Conclusions}

In this paper, we have reviewed the gradient-based edge detection methods on Spiral Architecture. The reviewed techniques include edge focusing, bilateral filter and triple-diagonal gradient. In order to improve the edge detection accuracy, an edge detection scheme combining the bilateral filter and triple-diagonal-gradient methods can be proposed. Bilateral filter can be used to better filter or suppress image noise while triple-diagonal-gradient can be applied for extraction of a clearer edge map.

\section{Acknowledgement}

This work is partly supported by the Australian Research Council Discovery Grant (DP0451666).

\section{REFERENCES}

Nalini K. Ratha, Tolga Acar, Muhittin Gokmen and Anil K. Jain, A distributed edge detection and surface reconstruction algorithm, Proc. Computer Architectures for Machine Perception, Como, Italy, 1995, pp.149-154.

G. Economou, S. Fotopoulos, and M. Vemis, A novel edge detector based on nonlinear local operations, Proc. IEEE International Symposium on Circuits and Systems, London, 1994, pp.293-296.

D. Marr and E. Hidreth, Theory of edge detection, Proc. Royal Soc., 1980, pp.187217.

R. M. Haralick, Edge and region analysis for digital image data, Computer Vision Graphics and Image Processing, 1980, pp.60-73.

J. F. Canny, A computational approach to edge detection, IEEE Transactions on Pattern Analysis and Machine Intelligence, Vol.8 1986, pp.679-698.

Phil Sheridan, Sprial architecture for machine vision, Ph.D. thesis, University of Technology, Sydney, 1996. 
Xiangjian He and Tom Hintz, Refining Edge Detection within Spiral Architecture, in Australian Computer Science Communications, Vol. 22, No.1, 2000, J.Edwards (ed.) pp.113-119, IEEE Computer Society Press, Washington.

Jun Zhou, Qimei Hu and Xiangjian He, Detection with Bilateral Filtering in Spiral Space, Proc. of The Second International Conference on Information Technology and Applications, Harbin, China, January, 2004, pp.222-225.

Qiang Wu, Xiangjian He and Tom Hintz, Bi-lateral Filtering Based Edge Detection on Hexagonal Architecture, Proc. 2005 IEEE International Conference on Acoustics, Speech, and Signal Processing, Philadelphia, PA, USA, Volume II, 2005, pp.713716.

Qiang Wu, Xiangjian He and Tom Hintz, A Triple-Diagonal Gradient-Based Edge Detection, 6th IASTED International Conference on Computers, Graphics, and Imaging (CGIM'2003), Honolulu, USA, August 2003, pp.244-249.

Wenjing Jia, Xiangjian He and Qiang Wu, Edge Analysis of Rectangular and Hexagonal Structures, Proc. International Conference of Information Communication Technology, Thailand, December 2004, pp.69-75.

Jon Sporring, Mads Nielsen, Luck Florack, and Peter Johansen, Gaussian scalespace theory, Kluwer Academic Publishers, 1997.

What lies behind us and what lies before us

are tiny matters

compared to what lies within us.

OLIVER WENDELL HOLMES 


\section{AT THE CENTER}

"In order to write a personal mission statement, we must begin at the very center of our Circle of Influence, that center comprised of our most basic paradigms, the lens through which we see the world.

It is here that we deal with our vision and our values. It is here that we use our endowment of self-awareness to examine our maps and, if we value correct principles, to make certain that our maps accurately describe the territory, that our paradigms are based on principles and reality. It is here that we use our endowment of conscience as a compass to help us detect our own unique talents and areas of contribution. It is here that we use our endowment of imagination to mentally create the end we desire, giving direction and purpose to our beginnings and providing the substance of a written personal constitution.

It is also here that our focused efforts achieve the greatest results. As we work within the very center of our Circle of Influence, we expand it. This is highest leverage PC (production capability) work, significantly impacting the effectiveness of every aspect of our lives.

Whatever is at the center of our life will be the source of our security, guidance, wisdom, and power.

Security represents your sense of worth, your identity, your emotional anchorage, your self-esteem, your basic personal strength or lack of it.

Guidance means your source of direction in life. Encompassed by your map, your internal frame of reference that interprets for you what is happening out there, are standards or principles or implicit criteria that govern moment by moment decision-making and doing. Wisdom is your perspective on life, your sense of balance, your understanding of how the various parts and principles apply and relate to each other. It embraces judgment, discernment, comprehension. It is a gestalt or oneness, an integrated wholeness.

Power is the faculty or capacity to act, the strength and potency to accomplish something. It is the vital energy to make choices and decisions. It also includes the capacity to overcome deeply embedded habits and to cultivate higher, more effective ones.

These four factors - security, guidance, wisdom, and power - are interdependent. Security and clear guidance bring true wisdom, and wisdom becomes the spark or catalyst to release and direct power. When these four factors are present together, harmonized and enlivened by each other, they create the great force of a noble personality, a balanced character, a beautifully integrated individual."

Stephen R. Covey 